\title{
Designing a virtual domotic system applied in educational environments
}

\author{
Chadwick Carreto $^{1}$, Elena F.Ruiz ${ }^{1}$, Marina Vicario ${ }^{2}$ \\ ${ }^{1}$ Escuela Superior de Cómputo - Instituto Politécnico Nacional. \\ Av. Juan de Dios Bátiz s/n Unidad Profesional "Adolfo López Mateos" \\ C.P. 07738, México D.F.Tel. (5255)5729-6000 Ext. 52051 \\ ${ }^{2}$ Unidad Politécnica Interdisciplinaria de Ciencias Sociales y Administrativas \\ Av. Instituto Politécnico Nacional s/n, Unidad Profesional "Adolfo López Mateos", \\ Edif. 1, 2, 3, 4 y 5, Col. Lindavista, México, D.F. C.P. 07738 \\ ${ }^{1}$ ccarretoa@ipn.mx, ${ }^{2}$ elen_fruiz@yahoo.com.mx, \\ ${ }^{3}$ marina.vicario@gmail.com
}

\begin{abstract}
In this paper the design of a Domotic Model applied on an educational setting, according to the proposed model, users can access environment seamlessly, anytime, anywhere shown by different mobile devices to information and services available within the different domains of work (administrative areas, classrooms, libraries, etc.). available to this environment. The proposed model seeks to be independent of the technology that is implemented (Wi-Fi, Bluetooth, etc..) And any network topology (wired, wireless, P2P, etc.) the proposed model fits the concept of Internet of Things and define a concept of "Virtual Domotic" beacuse extending the concept to larger workspaces and mobile.
\end{abstract}

\section{Introduction}

In the last years, the technological advance on domotic systems, mobile computing systems and wireless networks has been too relevant. These technologies make out an important basis in the current development schemes, so the provision of services for each technology represents a challenge for technological development, scientific and research communities.

Domotic means automation (provision of services for the management of resources, security, communications, comfort and leisure) within a limited and closed space. This concept has been classified according to the extension of the space that it can cover. There are three main elements into this classification: Domotic, which acts on the extension of small buildings as homes. Inmotic, that operates on the extension of buildings such as hospitals or offices, and Urbotic which unfolds on cities covering tertiary buildings and outdoor urbanization [1,2]. Mobile computation involves a simple concept: information process without wires, whereas the mobile device is not visibly connected to any computer. 
This concept includes two fundamental technological advances: wireless networks for data transmission (implemented on various wireless technologies such as $\mathrm{Wi}-\mathrm{Fi}$, Wimax, Bluetooth, etc.), and the miniaturization of computer device components that allows the development of more robust mobile devices, with a bigger capacity for processing (PDA's, Laptops, NoteBooks, SmartPhones, etc.). Their own portability has become them in a clothing accessory; an indispensable tool for every day.

Ubiquitous computing is based on the device perception capacity; it aims to build devices and highly sensitive systems that detect user's actions and environmental changes, in the same way as another person could do it. Once the user comes into the domain field, the devices may act according user's demand in order to provide him services for his requirements, in a totally transparent way [3]. Because of different aspects about the Administration and the security of the information, it becomes necessary to integrate and to divide networks into different topologies and administration logical distributions which aim a restructure in the global access and in the available services. By a Division of a network into domains (limited spaces, inside of which, there is some connection between devices) [4] [7], we'll have access to information that will be useful, because it will be focused on the staff and on the executed activity in such domains. This scheme of work claims to provide automatically several available services that are trying to deal with this administration at the level of communication protocols to improve the security of the system [5] [8].

Taking into account all these aspects, domotic, mobile computing and ubiquitous computing can provide several solutions to problems of diverse nature, which can range from simple consultation and/or information exchange to systems that allow users to collaborate from anywhere and at any time, so the main objective of this work is to develop a model, which will have as goal to offer to the educational environment user an access to the information and/or to the services of each work domain, in a transparent way from anywhere, at any time, providing him in this way, comfort within the educational environment. In the model proposed in this document, we take advantage of Virtual Domotic concept which let us out extend the original concept of Domotic, about automate certain limited space, taking the automation towards more spaces as it is the educational environment (all the buildings and physical facilities inside an educational campus) covering it completely.

Below, section 2 describes the model to develop. Section 3 explains model design and its development. Finally, in section 4, we give you a conclusion of the whole work and after that, we comment about future work.

\section{Virtual Domotic System Model}

The Virtual Domotic System Model (VDSM) (Fig. 1) is made up by modules of discovery, identification, services reporting, collection services and interaction network. The VDSM modules are described below: 
Designing a virtual domotic system applied in educational environments

\begin{tabular}{|c|c|c|c|c|}
\hline $\begin{array}{c}\text { Network } \\
\text { discovery } \\
\text { module. }\end{array}$ & $\begin{array}{l}\text { Identification } \\
\text { module. }\end{array}$ & $\begin{array}{c}\text { Presentation } \\
\text { services } \\
\text { module. }\end{array}$ & $\begin{array}{l}\text { Obtaining } \\
\text { services } \\
\text { module. }\end{array}$ & 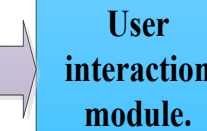 \\
\hline
\end{tabular}

Fig. 1. Virtual Domotic System Model

Network discovery module.- In this module, the user's mobile device detects the network or domain from the educational environment using access points, in order to start the communication between the device and the domain, where the access point perceives through the MAC address of the mobile device, if you are a user already registered in the database of the server.

Identification module.- This module performs validation on the server, by an identifier (password) that the user employs to register himself into the domain and in order to get access to the services. If the user's identification is positive, it proceeds to give him access to the domain and provide him the legal services.

Services Presentation module.- This module performs the services administration which each user accounts. It undertakes to present the services to the user in an organized and available way, when they are required. This module depends on the identification module, because in order to establish the services administration, the user needs previously require authentication.

Services Obtaining module.- This module implements the connection and the disconnection between the server and services domains. Also, the module presents depends on the identification module in order to establish the connection previously required for authentication by the user.

User interaction module.- It is the last of the modules and it is responsible for initializing the application by the user and display the response to that request, i.e. the denial or the delivery of information and/or services required according to the user's profile. This interaction should be simple, transparent and ubiquitous.

\section{VDSM Architecture}

For VDSM Architecture, we use the Unified Modelling Language (UML). Afterwards, based on the general model use case, we're going to establish the different states in which the VDSM will go through.

A) VDSM Design.

In order to satisfy with the expectative of the VDSM, we will divide the whole system in five use cases based on the VDSM modules (Figure 2). The user will interact directly with each one of use cases. 


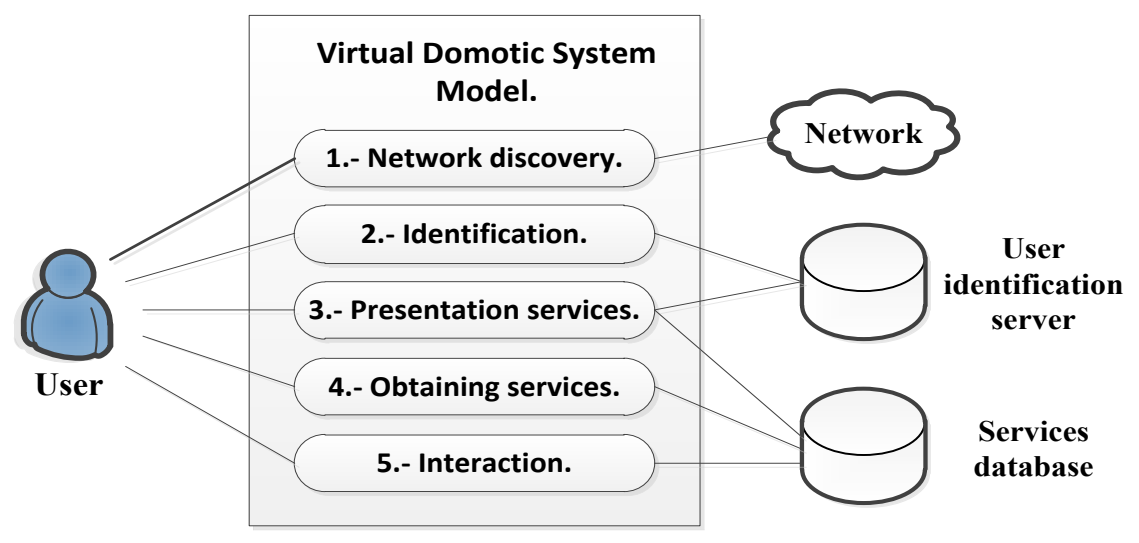

Fig. 2. Cases of use of the virtual domotic system model.

In the first case, user's computer identifies a network and it attempts to get access in order to receive the services that exist within it. The network assigns a valid address and links it with the next module.

The second case identifies the user. It refers to the ID module which identifies and authenticates the user. The authentication server will send the user an identification request. Then, the user will reply to the server and if it determines that the user answered correctly, the request will give him access to services according his preset profile. If the user does not fulfill the request, any access to the local network will be denied, so he'll get limited access.

The third case involves the service presentation module which is responsible for organizing the available services. In this way, the user can easily decide which service he'll use.

In the fourth case, the user requests a service according his profile which is previously identified. In this way, we set out and close the connection between the user and the services.

The final case is the interaction that the user has with the services. This one should be transparent and simple as possible each time the user requests information.

\section{VDSM Implementation testing}

VDSM architecture will be implemented within an educational environment, in order to ensure a functional model. Currently, we are pre-testing before take it to an educational environment. The network for this pre-testing is formed by:

- S.O.Ubuntu (VMWare) server.

- Access point Linksys WRT54G Wi-Fi 802. 11b/g (OS OpenWRT).

- S.O. Windows XP (user) Laptop Compaq

- An educational services server (AAVAE).

These are modular tests, so we have carried out as follows:

A) Network discovery module. 
First of all, we have configured a Linksys AP (model WRT54GS), where successfully, we have uploaded a Linux distribution called OpenWRT, which is able to program a Shell in charge to update each certain time, users connected list to the AP.

After that, we have developed in a Java language (within the server), a program that will be retrieving the users connected list to the AP. Also, it will be verifying in a database (within the server), the MAC address of the users connected to the AP. In this way, once a new user connected to the AP has already registered in the database, this module will pass control to the next one, in order to identify the user and give him access to the services according his profile.

B) Identification module.

According to the concept described in the 802.1X standard, we will implement an authentication server that will verify the authenticity of the user using the data that are sent because of the connection with the AP.

The new user is redirected to an HTML page where we inform him that it is necessary to carry out a prior authentication before access to the network services. To request data from the user, it establishes a secure communication, which presents to the user a template where should type the data required for authentication.

In this Authentication Server Module, we'll use RADIUS server to provide an authentication decision-making centralized mechanism.

C) Services Presentation Module.

In this module we are reusing the AAVAE system and its services (located on a services server) that are submitted to the user according to his profile, so he won't need to identify himself once again.

AAVAE (Fig. 3) is an architecture that will provide virtual educational services to IPN teachers and students, in order to interact in a synchronous and asynchronous mode, using tools such as streaming video, archives management, forums and chat.

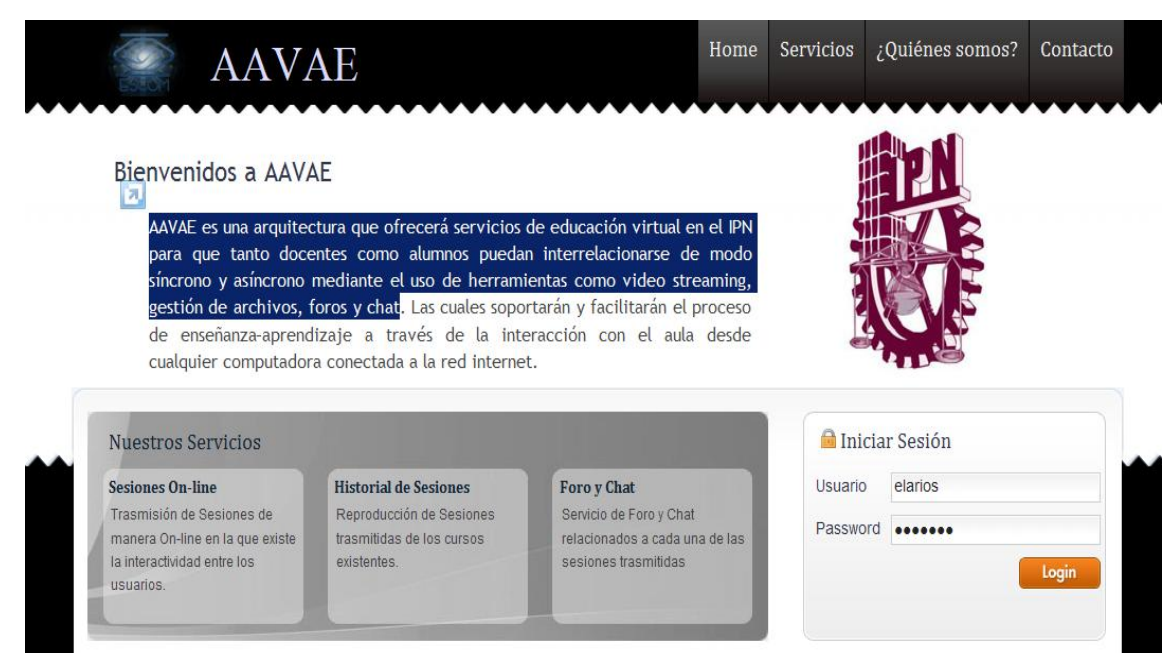

Fig. 3. AAVAE Educational Services System 
D) Services Obtaining Module.

This module tests the AAVAE system connection with the different repositories where the information and/or services are offered by this system to users, in order to be sure that this process will be in a transparent way.

E) Interaction module.

Fig. 4 shows the user interface. This will be simple for a no expert user, who will use without any problem, all the available services.

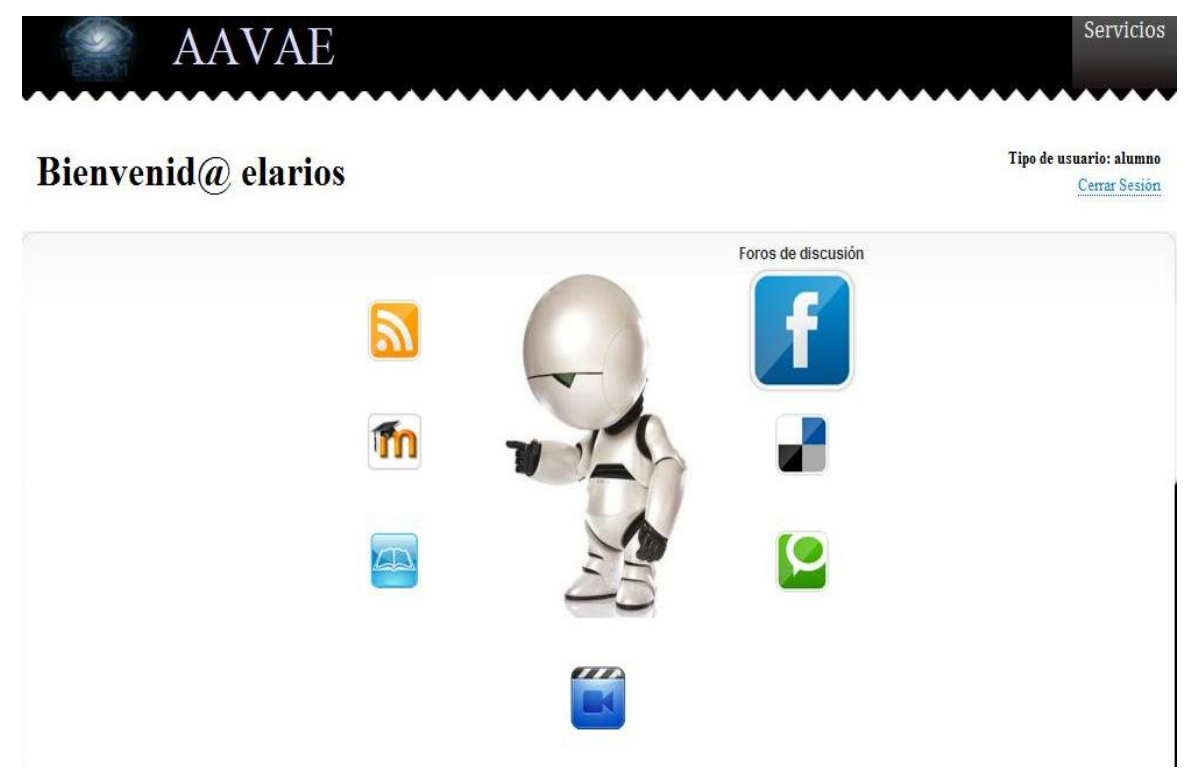

Fig. 4. Interaction Screen with AAVAE system's user

The user will only select one of the services offered on the screen, in order to have access to them. Through this system, the user will have one additional support tool for his education.

\section{Conclusions and future work}

The main contribution of the proposed model is to provide comfort to the users of the educational environment system, on the basis of mobility allowed by acting, given them saving time and effort in the access to the information and/or services.

Besides, this research helps us to extend Domotic concept toward a Domotic Virtual concept, because it not only covers certain areas or buildings within the environment, it contemplates the whole educational environment according to the different available domains.

Mobile networks are becoming more common in educational institutions. Nowadays, it becomes a mean objective to achieve a computing ubiquitous which ensure a total interactivity at any time and from everywhere. In this way, this project 
is focused on bring us closer to such objectives, attempting to develop an environment capable of providing the required services for a specific users, always in the most transparent way.

The system could be also a perfect basis to develop a ubiquitous computational environment anywhere that warrants it, establishing a protocol for the implementation of services by different mobile devices.

The application of this model is applied in many environments:

- Health. In this environment, it can be implemented in a hospital. For example, a patient can book a medical consultation by his cell phone, instead to make an appointment with the receptionist. Also, we can obtain information from a hospitalized patient. Furthermore, the doctor could review his daily agenda.

- Cultural. It can be implemented at museums where visitors will use their mobile devices to get information of locations, paintings, sculptures, exhibitions, etc.

Acknowledgements. The authors appreciate the support from CONACYT and Instituto Politécnico Nacional, particularly ESIME, ESCOM, UPICSA, CIC, SIP and COFAA for carrying out this work.

\section{References}

1. Nozick, J.: La maison intelligente. Editions du Moniteur, (1988)

2. Ramón, J., Millán, T., Huidobro, J.: Domótica: Edificios Inteligentes. Limusa, (2007)

3. Carreto, Ch., Menchaca, R.: Arquitectura de Colaboración mediante dispositivos Móviles Aplicada a la Administración del Conocimiento. TCM2004. ENC. Universidad de Colima, México (2004)

4. Muñoz, M.: Cómputo colaborativo consciente del contexto. Tesis de Maestría, CICESE, (2003)

5. Parsa, C., Garcia, J.: Improving TCP Performance over Wireless Networks at The Link Layer. ACM Mobile Networks and Applications Journal, 5(1), 57-71 (2000)

6. Murthy, S., Garcia, J.: A Routing Architecture for Mobile Integrated Services Networks. ACM Mobile Networks and Applications Journal. 3(4), 391-407 (1998)

7. Weiser: Ubiquitous computing. Intel Architecture Labs; (1994)

8. Weiser: Ubiquitous computing: origins, current research, and the future. Distinguished Lecture Symposium at University of British Columbia, Vancouver, British Columbia, Canada, (1993) 